\title{
Method Verification of Inhouse Real-time Polymerase Chain Reaction for Detection of Leishmania Species
}

\author{
Selma Usluca ${ }^{1}$ \\ ${ }^{1}$ General Directorate of Public Health, Microbiology Reference Laboratories and Biological Products Department, Ankara, Turkey
}

Received: 09 Novamber 2019, Accepted: 09 December 2019, Published online: 31 December 2019

(C) Ordu University Institute of Health Sciences, Turkey, 2019

\begin{abstract}
Objective: Leishmaniasis is a vector-borne disease caused by many Leishmania species which can infect both humans and other mammals. Turkey has special epidemiologic importance in terms of this disease due to being located at the junction of Asia and Europe and containing seven geographical regions with environmental and ecologic differences. Microscopic evaluation may miss diagnosis in cases with low levels of parasitemia. The culture method is not chosen by many laboratories due to being laborious preparation of media and results taking a long time. Molecular methods may assist in diagnosis especially with low parasitemia levels and provide results in a short time, so they have begun to be chosen more often currently. This study aimed to verify the method for inhouse real-time PCR using primers for ITS-1 gene region for routine molecular diagnosis of leishmaniasis.

Methods: After parasite counting from Leishmania infantum strain, which was carried out on NovyMacNeal-Nicolle (NNN) medium in our laboratory, DNA extraction was performed with a commercial kit (QIAamp DNA Blood Mini kit, Qiagen, Germany) according to the manufacturer's recommendations. The number of copies in the reaction was determined from DNA sample and serial dilutions of $1 / 10$ were prepared. Then, inhouse real-time PCR method was applied using primers targeting the ITS-1 gene region to determine limit of detection and high and low positivity rates. Then accuracy and precision studies were performed with these samples for method verification. Amplification was performed with a Light Cycler 96 (Roche, France) device. Results were evaluated with amplification curve analysis. The variation coefficients were calculated via accuracy and precision studies from the obtained results.

Results: The parasite counts of Leishmania infantum strain were determined as 17,000 promastigote/ml. With using $400 \mu 1$ of this sample $(6,800$ copies), DNA obtained in $100 \mu 1$ elution buffer. Using inhouse realtime PCR amplification, the detection limit for $2 \mu 1$ DNA (136 copies) was determined as $10^{-3}$ dilution $(0.136$ copies/reaction). The high positivity rate was determined as dilution above $2 \log 10$ of limit of detection's and the low positivity rate was determined as dilution above $1 \log 10$ of limit of detections. Efficiency of the method was measured with a regression curve for CT values. The standard curve obtained according to CT values and equivalent promastigote counts was linear (slope: -4.097). There was a significant correlation coefficient found between mean CT values and Leishmania infantum DNA concentrations $(\mathrm{R}=0.99)$.

Conclusion: Our study determined the variation coefficient for the inhouse real-time PCR method was below $15 \%$, confirming it is appropriate for use in our laboratory for routine molecular diagnosis of Leishmania spp.

Key words: Inhouse realtime PCR, method verification, Leishmania spp.
\end{abstract}

Suggested Citation: Usluca S. Verification of Inhouse Real-time Polymerase Chain Reaction for Detection of Leishmania Species. Middle Black Sea Journal of Health Science, 2019; 5(3): 192-198

Address for correspondence/reprints:

Selma Usluca

Telephone number: +90 (505) 2537123

E-mail: selmausluca@gmail.com

DOI: $10.19127 / \mathrm{mbsjohs.644748}$ 


\section{Introduction}

Leishmaniasis is a vector-borne disease caused by many Leishmania species which can infect both humans and other mammals (Galluzzi L, et al., 2018). It is accepted as one of the most common infectious diseases following HIV/AIDS, tuberculosis and malaria (Rezvan H, et al., 2017). According to the World Health Organization, more than 350 million people in 89 countries are at risk in terms of this disease (Rezvan, et al., 2017; Moreira, et al., 2018; Akhlagh, et al., 2019). Annually there are nearly 0.2 to 0.4 million visceral leishmaniasis cases and 0.7 to 1.2 million cutaneous leishmaniasis cases reported (Rezvan, et al., 2017; León, et al., 2017; Galluzzi, et al., 2018). Turkey has special epidemiologic importance in terms of this disease due to being located at the junction of Asia and Europe and containing seven geographical regions with environmental and ecologic differences (Ozensoy Toz, et al., 2013). Linked to Leishmania species and host features, the disease may occur with a variety of clinical appearances from asymptomatic infection, cutaneous leishmaniasis progressing with skin involvement, visceral leishmaniasis progressing with internal organ involvement, and mucocutaneous leishmaniasis progressing with mucosa and skin involvement (León, et al., 2017; Galluzzi, et al., 2018; Moreira, et al., 2018; Akhlagh, et al., 2019). Cutaneous leishmaniasis is the most commonly observed clinical form (Moreira, et al., 2018). There are two forms of cutaneous leishmaniasis: Anthroponotic cutaneous leishmaniasis caused by Leishmania tropica (L. tropica) as agent and zoonotic cutaneous leishmaniasis caused by Leishmania major (L. major) as agent (Rezvan, et al., 2017; Akhlagh, et al., 2019). In Turkey, anthroponotic cutaneous leishmaniasis caused by L. tropica is endemic in the southeast Anatolia, eastern Mediterranean and Aegean regions. In addition to $L$. tropica, in southeast Anatolia there are human cutaneous leishmaniasis cases reported caused by Leishmania infantum ( $L$. infantum) as agent. The disease is endemic in countries along Turkey's southern border (Syria, Iraq, and Iran) (Ozensoy Toz, et al., 2013). The most serious form of the disease is visceral leishmaniasis, also known as kala-azar. The etiologic agent is mostly $L$. infantum, and a Leishmania donovani (L. donovani) complex comprising L. donovani and Leishmania chagasi ( $L$. chagasi) (Rezvan, et al., 2017). In recent times, in addition to visceral leishmaniasis cases caused by $L$. tropica, cutaneous leishmaniasis cases caused by $L$. donovani and $L$. infantum are reported. Due to increased travel possibilities, climate changes, and changes in socioeconomic and sociopolitical conditions, new species may be observed in our country (Sirekbasan S, et al., 2109). Visceral leishmaniasis is endemic to the Mediterranean, Aegean, Marmara and Black Sea regions, while sporadic cases are observed in other regions (Ozensoy Toz S, et al., 2013). The mucocutaneous form (espundia) occurs due to Leishmania brazilensis (L. brazilensis), Leishmania panamensis (L. panamensis), and Leishmania guyanensis ( $L$. guyanensis) species in the New World, while frequently $L$. infantum and $L$. donovani species are agents in other regions. The most significant symptoms of cutaneous leishmaniasis is ulcerative skin lesions in bite region and multiple nonulcerative nodules. The symptoms of visceral leishmaniasis include fever, skin pigmentation, loss of appetite, fatigue, hepatosplenomegaly, lymphadenopathy, pancytopenia, weight loss and death. Due to showing similarities to many diseases, differential diagnosis is very important. While cutaneous leishmaniasis may be confused with leprosy, skin tuberculosis and malignancies, visceral leishmaniasis may be confused with malaria and schistosomiasis (Rezvan, et al., 2017).

\section{Methods}

After parasite counting from $L$. infantum strain, which was carried out on Novy-MacNeal-Nicolle (NNN) medium in our laboratory, DNA extraction was performed with a commercial kit (QIAamp DNA Blood Mini kit, Qiagen, Germany) according to the manufacturer's recommendations. The number of copies in the reaction was determined from DNA sample and serial dilutions of 1/10 were prepared. Then, inhouse real-time PCR method was applied using primers targeting the ITS-1 gene region (Table 1) to determine limit of detection and high and low positivity rates. The PCR mix rates for inhouse real-time PCR method are given in Table 2 and amplification conditions are given in Table 3. Then accuracy and precision studies were performed on these samples for method verification. As negative control, RNAase/DNAse free water was used. For qualitative tests, the accuracy studies was performed with three high and low positive and negative samples. Precision studies were applied in two ways, inter assay and intra assay study. While in intra assay precision studies, three high and three low positive samples were studied at one time, in inter assay precision studies one high and one low positive samples were studied at three different days. Amplification was performed with Light 
Cycler 96 (Roche, France) device. The results were evaluated with amplification curve analysis. The variation coefficients $(\mathrm{CV} \%)$ were calculated in accuracy and precision studies from the obtained results. For evaluation of method verification results, Cumitech 31A 2009 was taken as reference (Clark, et al., 2009).

Table 1. Inhouse real-time PCR primers

Forward primer 5'-CATTTTCCGATGATTACAC-3' LSGITS1-F1

Reverse primer 5'-CGTTATGTGAGCCGTTATC-3' LSGITS1-R1

Table 2. Inhouse real-time PCR mix

\begin{tabular}{lc}
\hline $\mathrm{H}_{2} \mathrm{O}$ & $6 \mu \mathrm{l}$ \\
\hline Syber Green Master mix & $10 \mu \mathrm{l}$ \\
\hline Primer F (LSGITS1-F1) & $1 \mu \mathrm{l}$ \\
\hline Primer R (LSGITS1-R1) & $1 \mu \mathrm{l}$ \\
\hline DNA & $2 \mu \mathrm{l}$ \\
\hline Total volume & $20 \mu \mathrm{l}$
\end{tabular}

Table 3. Amplification conditions of inhouse real-time PCR

\begin{tabular}{|c|c|c|}
\hline $95{ }^{0} \mathrm{C}$ & $5 \mathrm{~min}$ & $\begin{array}{l}\text { Initial } \\
\text { denaturation }\end{array}$ \\
\hline $95^{0} \mathrm{C}-15 \mathrm{sec}$ & \multirow{3}{*}{45 cycles } & \\
\hline $55^{0} \mathrm{C}-40 \mathrm{sec}$ & & \\
\hline $72{ }^{0} \mathrm{C}-60 \mathrm{sec}$ & & \\
\hline $72{ }^{0} \mathrm{C}$ & $2 \min$ & Final extention \\
\hline
\end{tabular}

\section{Results}

The parasite counts of $L$. infantum strain were determined as 17,000 promastigote/ml. With using $400 \mu 1$ of this sample (6,800 copies), DNA obtained in $100 \mu \mathrm{l}$ elution buffer. The study results for inhouse real-time PCR serial dilutions are given in Table 4. Using inhouse real-time PCR amplification, the limit of detection for $2 \mu 1$ DNA
(136 copies) was determined as $10^{-3}$ dilution $(0.136$ copies/reaction). The high positivity rate was determined as dilution above $2 \log 10$ of limit of detection's and the low positivity rate was determined as dilution above $1 \log 10$ of limit of detection's. The verification study results for inhouse real-time PCR method are presented in Table 5. To calculate variation coefficient, firstly standard deviation and mean were calculated, then coefficient of variation (CV\%) was obtained by dividing standard deviation to mean. Accordingly, when variation coefficients are calculated for both accuracy and precision studies, it was determined to be below $15 \%$. The amplification curves for limit of detection study of inhouse real-time PCR method are given in Figure 1. Efficiency of the method was measured with a regression curve for CT values. The standard curve obtained according to $\mathrm{CT}$ values and equivalent promastigote counts was linear (slope: -4.097) (Figure 2). There was a significant correlation coefficient between mean $\mathrm{CT}$ values and L. infantum DNA concentrations $(\mathrm{R}=0.99)$.

Table 4. Serial dilution results of inhouse real-time PCR

\begin{tabular}{ll}
\hline Dilution Rate & Copy number \\
\hline $10^{0}$ dilution & 136 copy \\
\hline $10^{-1}$ dilution (high positive) & 13,6 copy \\
\hline $10^{-2}$ dilution (low positive) & 1,36 copy \\
\hline $\mathbf{1 0}^{-3}$ dilution (limit of detection) & $\mathbf{0 , 1 3 6}$ copy \\
\hline
\end{tabular}

Table 5. The results of inhouse real-time PCR method verification study

\begin{tabular}{lccc}
\hline & $\begin{array}{c}\text { Accuracy } \\
\text { Study }\end{array}$ & $\begin{array}{c}\text { Precision Study } \\
\text { (intra assay) }\end{array}$ & $\begin{array}{c}\text { Precision } \\
\text { Study (inter } \\
\text { assay) }\end{array}$ \\
\hline $\begin{array}{l}\text { High } \\
\text { positive } \\
(\% \mathbf{C V})\end{array}$ & 0 & 0,0088 & 0,0230 \\
\hline $\begin{array}{l}\text { Low } \\
\text { positive } \\
(\% \mathrm{CV})\end{array}$ & 0 & 0,0136 & 0,0136 \\
\hline
\end{tabular}

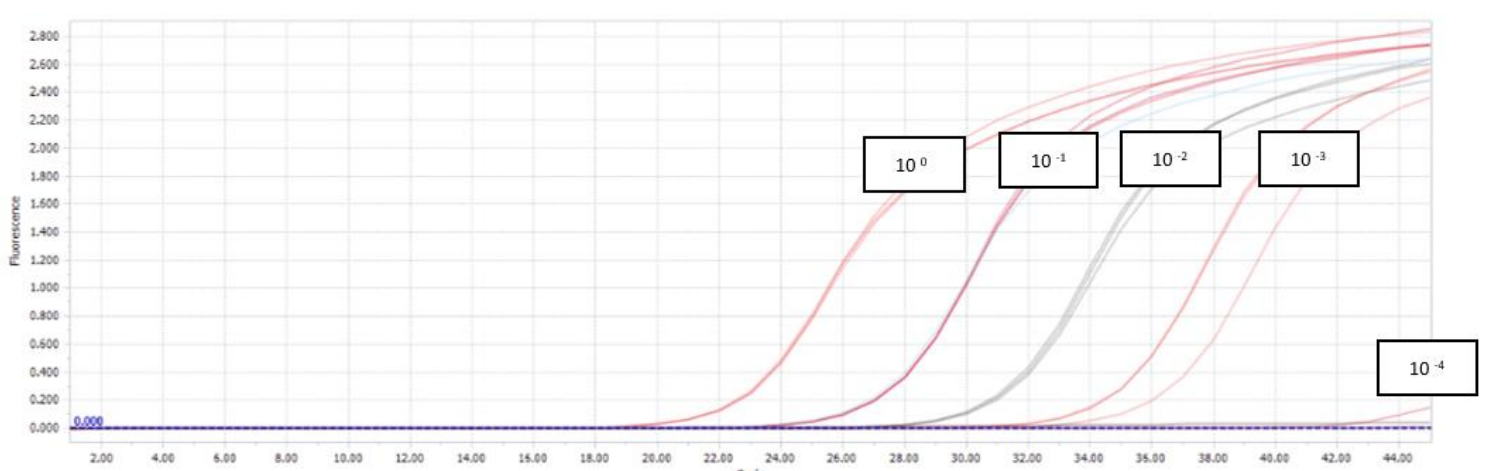

Figure 1. Amplification curves of limit of detection study of the inhouse real-time PCR method 


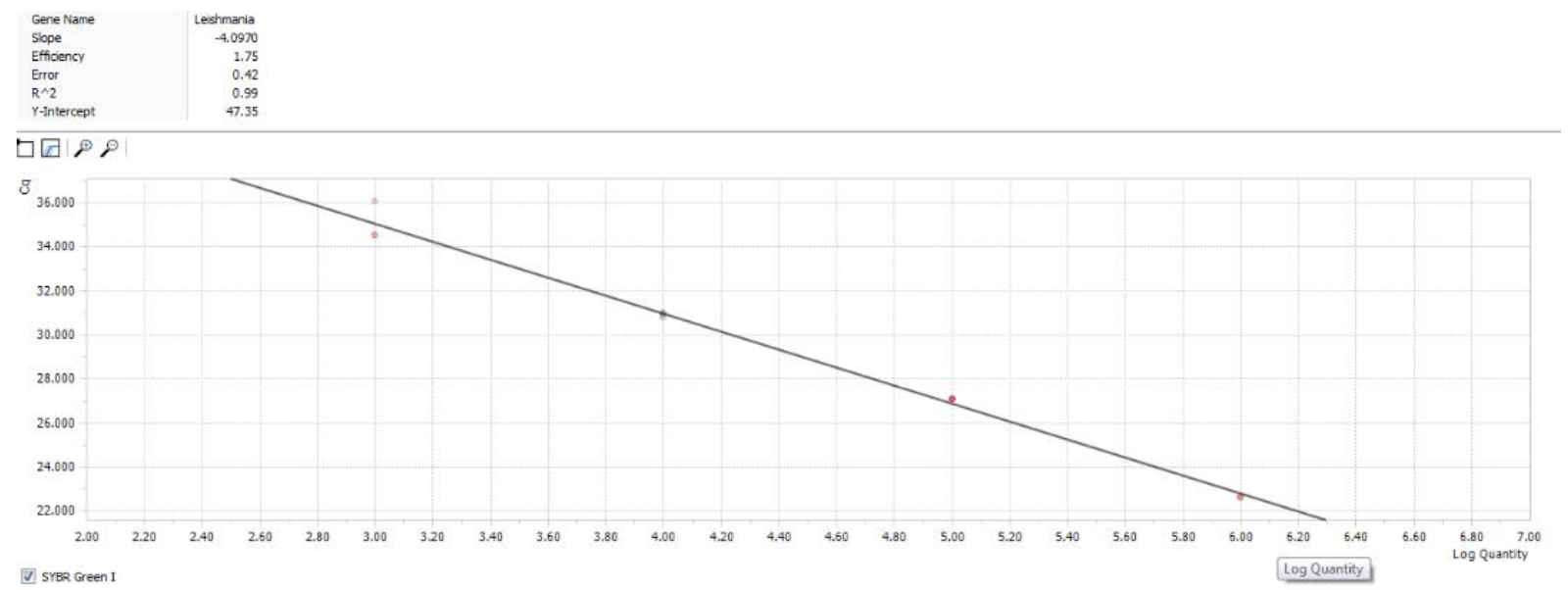

Figure 2. Plot of threshold cycle (CT) versus log promastigote number obtained from inhouse real-time PCR

\section{Discussion}

For diagnosis of the disease microscopy, culture, serologic and molecular methods are used (Mary, et al., 2004; Rezvan, et al., 2017; Moreira, et al., 2018; Soofi Abadi, et al., 2019; Deepachandi, et al., 2019). Microscopy is still accepted as the gold standard method. Parasite culture with NNN media may be used for supporting diagnosis. However, preparation of media is laborious, requires technical expertise, tends toward contamination, and it takes a long time (de Vries, et al., 2015; Deepachandi, et al., 2019). The sensitivity of parasite culture and accompanying microscopic investigation is reported to increase to up to $85 \%$ (Rezvan, et al., 2017). With the light microscopy, Leishmania species cannot be distinguished morphologically from each other; however, species differentiation is very important for patient management (de Vries, et al., 2015; Rezvan, et al., 2017). Additionally, the use of light microscopy with in vitro culture methods is limited for chronic, atypical or treated lesions (Deepachandi, et al., 2019). Though the specificity for microscopic investigation in routine diagnosis of cutaneous leishmaniasis especially is high (100\%), but sensitivity is low. The sensitivity of the method varies from $40-74.4 \%$, depending on condition of skin lesion from which sample is taken, localization from which sample is taken and expertise of personnel performing microscopic examination. As a result, more sensitive methods such as PCR were developed as alternatives for diagnosis of this disease (Rezvan, et al., 2017; León, et al., 2017). The serologic diagnosis of visceral leishmaniasis is based on methods such as indirect fluorescent antibodies, ELISA, western blot, lateral flow and direct agglutination test. However, these methods are not widely used for cutaneous leishmaniasis diagnosis due to weak humoral response caused by infection and low sensitivity (de Vries, et al., 2015). Molecular techniques such as PCR and real-time PCR can distinguish parasite species (Soofi Abadi MF, et al., 2019). Definition of parasite species is very important to understand the disease epidemiology (Ozensoy Toz, et al., 2013). Previously, isoenzyme analysis was used for species differentiation. This method is based on differences in electrophoretic mobilities of enzymes isolated from Leishmania species. However, due to high cost, time required and need for large amounts of cultured promastigote, it is only applied in a few reference laboratories. As a result, alternative typing methods have been developed especially based on the genetic features of the parasite (de Vries, et al., 2015; Rezvan, et al., 2017). Molecular methods are reported to have better sensitivity and specificity that traditional diagnostic methods (de Vries, et al., 2015). Methods such as conventional single-stage PCR, nested PCR, PCR-RFLP and quantitative real-time PCR (qPCR) are commonly used. Though nested PCR is one of the most sensitive PCR techniques, an important limitation is false positivity due to possible cross contamination (Hernández, et al., 2014; Deepachandi, et al., 2019). QPCR is a method that is more rapid compared method to traditional PCR, repeatable, has high sensitivity and allows quantitative testing (PaivaCavalcanti, et al., 2010; Hernández, 2014; Galluzzi, et al., 2018; Moreira, et al., 2018; Antonia, et al., 2018). As reaction tubes do not need to be opened after amplification, cross contamination is largely reduced (Galluzzi, et al., 2018). Real-time PCR is based on use of stains or fluorescent probes allowing observation of the amplified product (Paiva-Cavalcanti, et al., 2010; Galluzzi, et al., 2018). With this aim, SYBR Green is a commonly used stain and binds nonspecifically to DNA double 
strand produced during amplification (PaivaCavalcanti, et al., 2010). SYBR Green based analyses and HRM analyses allow possibility of differentiating Leishmania subgenus or species according to melting curves obtained for each sample (Moreira, et al., 2018). Another way to produce fluorescence is to use a probe targeting the internal sequence region required for amplification (e.g., TaqMan probe). During amplification TaqMan degrades and leaves a light-emitting reporter. Light emission analysis is made by a light signal detector forming a graph with absorbance obtained from each PCR cycle. The produced signal reflects the amount of product produced (PaivaCavalcanti, et al., 2010). In terms of cost, Taqman analysis has higher cost. Additionally, due to allowing the possibility of identifying multiplex reactions, the test costs may be accepted as relatively lower (Moreira, et al., 2018). The point when the fluorescence emission of sample exceeds a threshold value is called cycle threshold (CT) and is related to amount of amplified DNA. Along the amplification curve, the $\mathrm{CT}$ of each analyzed sample can be checked and decisions made about whether it is positive or not. Values above 35 CT may cause false positive results (Paiva-Cavalcanti, et al., 2010). The reasons for this situation are considered to be samples containing an inhibitor or low parasitemia levels. After treatment, a similar situation may be encountered with samples. As a result, evaluations should be made carefully. For example, the inhibitor content problem may be solved by studying diluted DNA, while the increase in DNA amounts in samples with low parasitemia rates may reduce $\mathrm{CT}$ values. The evaluation results in our study accepted samples with CT value above 38 as being negative. The sensitivity of molecular methods varies linked to the selection of target gene region (Mary, et al., 2004; Galluzzi, et al., 2018). With this aim, many target genes have been defined. The most commonly used multiple copy genes such as kDNA, ITS-1 and HSP70 genes have advantages such as low detection limits and potential to differentiate species (Hernández, et al., 2014; de Vries, et al., 2015; Rezvan, et al., 2017; Moreira, et al., 2018; Antonia, et al., 2018). KDNA can be determined up to 10,000 copies, 18S rRNA has 166 copies, ITS- 1 has 40 to 200 copies and HSP70 has 5 to 7 copies. When evaluated in terms of detection limits, multiple copy genes may reveal significant degrees of variability between parasite species, strains, life cycle stages and clinical isolates. Single copy genes tend to be found in more stable regions of genome; however, detection limits are generally higher compared to multicopy genes. Single copy genes are glucose phosphate isomerase (GPI), glucose 6 phosphate dehydrogenase (G6PD), superoxide dismutase 1 (SODB1), arginine permease (AAP3) and DNA polymerase (Antonia, et al., 2018). Though there are many studies published about qPCR, each laboratory applies their own method's as there are no determined general protocols. There are very few studies about comparisons between laboratories, especially. There is requirement for studies on this topic (de Vries, et al., 2015). Additionally, evaluation of analytic performance to confirm a diagnostic method requires determination of repeatability, accuracy and detection limits (León, et al., 2017; Soofi Abadi, et al., 2019). Determination of parasite amounts with qPCR can be performed with definite or relative quantitation. For quantification a standard curve obtained with purified parasite genomic DNA or target sequence's serial dilutions is used. With this data, target DNA found in a sample can be determined according to known DNA amount (Paiva-Cavalcanti, et al., 2010; Galluzzi, et al., 2018). In our laboratory we performed verification of the inhouse real-time PCR method with primers targeting ITS-1 gene region used for routine molecular diagnosis of leishmaniasis. With this aim, after determining the limit of detection by applying real-time PCR to $1 / 10$ serial dilutions of DNA obtained from $L$. infantum strains growing in NNN media in our laboratory, we completed method verification studies. The results of our study showed the detection limit for our method was 0.136 copies/reaction, which is in accordance with the literature. When detection limit for target gene regions is evaluated, qPCR detection limit for $\mathrm{kDNA}$ and $18 \mathrm{~S}$ primers was $1 \times 10^{-2}$ parasite $/ \mathrm{ml}$, and qPCR detection limit for HSP70 and ITS-1 primers was reported as $1 \times 10^{-1}$ parasite/ml (León, et al., 2017). Another study reported detection limit for real-time PCR method with kDNA was 0.0125 parasite DNA/ml (Mary, et al., 2004).

The obtained standard curve also provides curve used to calculate amplification efficiency. For each 10 dilutions of the target, a slope of-3.32 represents $100 \%$ efficiency and shows the amplified molecule numbers increase two times with each PCR cycle (Paiva-Cavalcanti M, et al., 2010 While slopes that are more negative than-3.32 (e.g.,-3.9) show efficiency lower than $100 \%$, slopes more positive than -3.32 (e.g., -2.5) show problems with sample quality or pipetting (ABI_Guide_Relative_ Quantification_using_realtime_PCR). A variety of studies reported standard curves from-3.23 to-3.40 
(Mary, et al., 2004; Soofi Abadi, et al., 2019). The slope of curve obtained in our study was determined as-4.097. This situation shows efficiency is lower than $100 \%$; however, it is acceptable and indicates our DNA quality was good and there were no pipetting errors when preparing dilutions. The correlation coefficient appears to be between 0.996 to 0.998 in a various studies (Mary, et al., 2004; Hossain, et al., 2017; Soofi Abadi, et al., 2019). Similarly, correlation coefficient obtained at the end of our study was determined as 0.99 .

\section{Conclusion}

In our study variation coefficient for inhouse real-time PCR method was below $15 \%$, efficiency of the method was determined to be sufficient and decision was made that it is appropriate for use for routine molecular diagnosis of Leishmania spp. in our laboratory.

Peer-review: Externally peer-reviewed.

Author Contributions: Concept - SU Design SU; Supervision SU; Materials - SU; Data Collection and/or Processing - SU; Analysis and/or Interpretation - SU; Literature Review - SU; Writing - SU; Critical Review - SU

Conflict of Interest: No conflict of interest was declared by the author.

Financial Disclosure: The author declared that this study hasn't received no financial support.

\section{References}

Akhlagh A, Salehzadeh A, Zahirnia AH and Davari B, 10-Year Trends in Epidemiology, Diagnosis, and Treatment of Cutaneous Leishmaniasis in Hamadan Province, West of Iran (2007-2016), Front Public Health, 2019; 7:27.

Antonia AL, Wang L, Ko DC. 2018. A Real-time PCR Assay for Quantification of Parasite Burden in Murine Models of Leishmaniasis, Peer J, 6:e5905: 1-17.

Clark RB, Lewinski MA, Loeffelholz MJ, Tibbetts RJ, 2009. Cumitech 31A, verification and validation of procedures in the clinical microbiology laboratory. Coordinating ed., S. E. Sharp. ASM Press, Washington, DC.

Deepachandi B, Weerasinghe S, Soysa P, Karunaweera N, Siriwardana Y, A Highly Sensitive Modified Nested PCR to Enhance Case Detection in Leishmaniasis, BMC Infect Dis, 2019; 19:623.

de Vries HJC, Reedijk SH, Schallig HDFH, Cutaneous Leishmaniasis: Recent
Developments in Diagnosis and Management, Am J Clin Dermatol, 2015;16: 99-109.

Galluzzi L, Ceccarelli M, Diotallevi A, Menotta M, Magnani M, Real-time PCR applications for diagnosis of leishmaniasis, Parasites \& Vectors, 2018;11(273): 1-13.

Hernández C, Alvarez C, González C, Ayala MS, León CM, Ramírez JD, Identification of Six New World Leishmania species through the implementation of a High-Resolution Melting (HRM) genotyping assay, Parasit Vectors, 2014; 7: 501, p. 1-7.

Hossain F, Ghosh P, Khan M.AA, Duthie MS, Vallur AC, Picone A, Howard RF, Reed SG, Mondal D, Real-time PCR in Detection and Quantitation of Leishmania donovani for the Diagnosis of Visceral Leishmaniasis Patients and the Monitoring of Their Response to Treatment, PLoS ONE, 2017;12(9): e0185606. https://www.gu.se/digitalAssets/1125/1125331_A

BI_Guide_Relative_Quantification_using_realti me_PCR.pdf

León CM, Muñoz M, Hernández C, Ayala MS, Flórez C, Teherán A, Cubides JR, Ramírez JD, Analytical Performance of Four Polymerase Chain Reaction (PCR) and Real Time PCR (qPCR) Assays for the Detection of Six Leishmania Species DNA in Colombia, Front Microbiol, 2017;8(1907): 1-13.

Mary C, Faraut F, Lascombe L, Dumon H, Quantification of Leishmania infantum DNA by a Real-Time PCR Assay with High Sensitivity, J Clin Microbiol, 2004;42(11): 5249-5255.

Moreira OC, Yadon ZE, Cupolillo E, The applicability of real-time PCR in the diagnostic of cutaneous leishmaniasis and parasite quantification for clinical management: Current status and perspectives, Acta Tropica, 2018;184: 29-37.

Ozensoy Toz S, Culha G, Yildiz Zeyrek F, Ertabaklar H, Alkan MZ, Tetik Vardarlı A, Gunduz C, Ozbel Y, A Real-Time ITS1-PCR Based Method in the Diagnosis and Species Identification of Leishmania Parasite from Human and Dog Clinical Samples in Turkey, PLoS Negl Trop Dis, 2013;7(5): e2205.

Paiva-Cavalcanti M, Regis-da-Silva CG, Gomes YM, Comparison of Real-time PCR and Conventional PCR for Detection of Leishmania (Leishmania) infantum Infection: A MiniReview, J Venom Anim Toxins incl Trop Dis, 2010;16(4): 537-542. 
Rezvan H, Nourian AR, Hamoon Navard S, An Overview on Leishmania Diagnosis, J Med Microbiol Infec Dis, 2017;5 (1-2): 1-11.

Sirekbasan S, Polat E, Kutlubay Z, Engin B, Leishmania infantum'un Etken Olduğu Bir Kutanöz Leishmaniasis Olgusu, Türkiye Parazitol Derg, 2019;(43)1:41-3.

Soofi Abadi MF, Fekri M, Moradabadi AR, Vahidi R, Shamsi-Meymandi S, Dabiri D, Dabiri S, Ability of Real-time PCR for Differential Diagnosis of Various Forms of Cutaneous Leishmaniasis: A Comparative Study with Histopathology, BMC Res Notes, 2019; 12:615.365. 